\title{
Caracterización del origen del ligamento suspensorio en miembro anterior de equinos mediante ultrasonido y resonancia magnética
}

\author{
Pirota, V.L. ${ }^{1}$; Lértora, W. J. ${ }^{1}$; Forlino, D. ${ }^{2}$ \\ Cátedras de Anatomía y Patología, Facultad Cs.Veterinaria, UNNE ${ }^{1}$ \\ Cátedra de Anatomía Humana, Facultad de Medicina, UNNE ${ }^{2}$ \\ Corrientes, Argentina. E-mail: veronicapirota $(a$ hotmail.com
}

\begin{abstract}
Resumen
Pirota, V.L.; Lértora, W.J.; Forlino, D.: Caracterización del origen del ligamento suspensorio en miembro anterior de equinos mediante ultrasonido y resonancia magnética. Rev. Vet. 32: 2, 181-184, 2021. El objetivo del estudio fue describir la anatomía normal del origen del ligamento suspensorio del miembro anterior de equinos de trabajo, mediante el análisis de ultrasonido y resonancia magnética. Se examinaron clínicamente equinos de trabajo destinados a un frigorífico, constatando ausencia de lesiones en el ligamento suspensorio de miembros anteriores. Luego se realizó el análisis mediante ultrasonido y posteriormente a la faena se procedió al examen de resonancia magnética con las piezas cadavéricas previamente acondicionadas. Se obtuvieron imágenes transversales y longitudinales por ultrasonido. Con resonancia magnética se adquirieron imágenes longitudinal, transversal y dorsal. En ambos métodos diagnósticos se lograron medidas del diámetro dorso-palmar y latero-medial. Las imágenes en corte transversal de ultrasonido demostraron la forma rectangular del ligamento suspensorio y en imágenes longitudinales se observó el característico alineamiento fibrilar del ligamento. En cuanto a la evaluación mediante resonancia magnética, en imágenes transversales se visualizó una forma bilobulada del ligamento con una zona central oscura, y una periférica blanca debido a las intensidades de señal que adoptaron los diferentes tejidos que lo conformaron. En la imagen longitudinal se vio un aspecto de banda lisa y uniforme. En la imagen dorsal se visualizaron como líneas verticales, siendo la intensidad de la señal de intermedia a alta. La ultrasonografía y la resonancia magnética nos permitieron evaluar la constitución tisular variada que presentó el origen del ligamento suspensorio.
\end{abstract}

Palabras clave: equinos, resonancia magnética, ultrasonografía, ligamento suspensorio

\begin{abstract}
.
Pirota, V.L.; Lértora, W.J.; Forlino, D.: Characterization of the origin of the suspensory ligament in the forelimb working horses by magnetic resonance and ultrasound. Rev. Vet. 32: 2, 181-184, 2021. The aim of study was to describe normal anatomy of the origin of the suspensory ligament in the forelimb working horses by ultrasound and resonance magnetic Working horses headed to a slaughter house were clinical examined, they were verified with absence injuries in the suspensory ligament in the forelimbs. Then ultrasound analysis was done and subsequently to the slaughter, resonance magnetic analysis with pieces cadaverous previously packed were done. Transverse and longitudinal images were obtained by ultrasound and with magnetic resonance longitudinal, transverse and dorsal images. In both diagnostic methods were obtained dorsal-palm and lateral-medial diameter measurements. Cross-sectional images demonstrated the rectangular shape of the suspensory ligament and longitudinal images characteristic fibrilar alignment of the ligament was observed. Regarding evaluation by magnetic resonance, in cross-sectional images a billobed shape of the ligament was visualized with a dark central zone and a white peripheral zone due to the signal intensities adopted by the different tissues that comprise it. In the longitudinal image smooth and uniform band appearance. In the dorsal image it was visualized as vertical lines, the intensity signal being from intermediate to high. Ultra sonography and magnetic resonance imaging allowed us to evaluate the varied time constitution that the origin of the suspensory ligament presented.
\end{abstract}

Key words: horses, magnetic resonance, ultrasound, suspensory ligament. 


\section{INTRODUCCIÓN}

El ligamento suspensorio (LS), también conocido como músculo interóseo, discurre a lo largo del surco metacarpiano formado por la superficie palmar del tercer metacarpiano y las superficies axiales del segundo y cuarto metacarpianos, en los cuatro miembros del caballo. Se origina en la cara palmar de la extremidad proximal del hueso metacarpo III y huesos carpianos III y IV ${ }^{1,2}$.

Algunas fibras se fijan en la cara palmar de los huesos metacarpianos II y IV y toman contacto con el receso palmar de la articulación carpo-metacarpiana ${ }^{3}$. Está compuesto normalmente por una variable cantidad de fibras tendinosas, musculares y grasa ${ }^{2,3}$.

Desde una perspectiva funcional el LS soporta y evita la hiper extensión de la articulación metacarpo falangiana y metatarso falangiana en conjunto con otros ligamentos ${ }^{4}$. Su compleja arquitectura y ubicación determinan una alta prevalencia de lesiones que se localizan en el origen ${ }^{4-9} \mathrm{o}$ en su parte proximal ${ }^{3,5}$

La exploración clínica del LS y la utilización del ultrasonido (US) son los procedimientos más frecuentes en la práctica clínica para determinar su anatomía normal y patología. El US se emplea habitualmente como método de diagnóstico complementario a la exploración clínica para la detección de lesiones de diferentes estructuras músculo-esqueléticas del aparato locomotor del equino ${ }^{13-18}$.

Numerosos especialistas ${ }^{3,4-7,19,20}$ afirman que es esencial la aplicación del US para la observación normal del LS y el diagnóstico de una lesión. Con el avance de la tecnología se ha propuesto el uso de la resonancia magnética (RM) conjuntamente con la US. Los autores mencionados consideran el empleo de RM en aquellas lesiones que no pueden ser visualizadas con la US y en el diagnóstico de la remodelación ósea de la entesis del ligamento ${ }^{4-7}$.

Los trabajos publicados sobre la anatomía normal y patología del LS mediante US y RM fueron realizados en equinos deportivos, pero no se encuentran des- cripciones en caballos de trabajo. Por lo expuesto, el objetivo del trabajo fue describir la anatomía normal del origen del LS en miembros anteriores de equinos de trabajo asintomáticos mediante exámenes US y RM

\section{MATERIAL Y MÉTODOS}

Se estudiaron 12 miembros anteriores de equinos de raza indefinida, 7 machos y 5 hembras, adultos y clínicamente sanos. Los equinos eran procedentes de un establecimiento ubicado en Basail (Provincia del Chaco, Argentina) destinados aun frigorífico. Previo a la faena, se realizó el examen clínico a fin de constatar ausencia de lesiones en el LS y se identificó cada miembro con precintos.

Posteriormente, se realizó el examen de US del origen del LS, a $2 \mathrm{~cm}$ distal de la articulación del carpo, según lo propuesto por investigadores ${ }^{15}$, con un equipo Mindray Dp30 y transductor lineal 7,5 Mhz. La frecuencia, foco, profundidad y ganancia fueron ajustados para maximizar la calidad de las imágenes. Se obtuvieron imágenes transversales-axiales y longitudinalessagitales, y se registró el espesor dorso-palmar (D-P) y latero-medial (L-M) del LS.

Las RM se realizaron con las piezas anatómicas, previamente fijadas en formol al $10 \%$ en bolsas herméticas, y se utilizó un equipo de 1.5 Tesla de aplicación clínica general con una bobina de rodilla de 8 canales. Se obtuvieron secuencias de imágenes en $\mathrm{T} 1$, o de relajación transversal, T2 o de relajación longitudinal y de densidad protónica en los planos sagital-longitudinal, axial-transversal y coronal-dorsal. Se registró el espesor dorso-palmar (D-P) y latero-medial (L-M) del LS.

Las datos del espesor DP y LS se expresaron en centímetros, en US y RM, y se realizó el análisis de las medias mediante el programa InfoStat (2008).

\section{RESULTADOS}

El origen del LS adoptó una forma rectangular en los cortes transversales (Figura 1) y su composición de
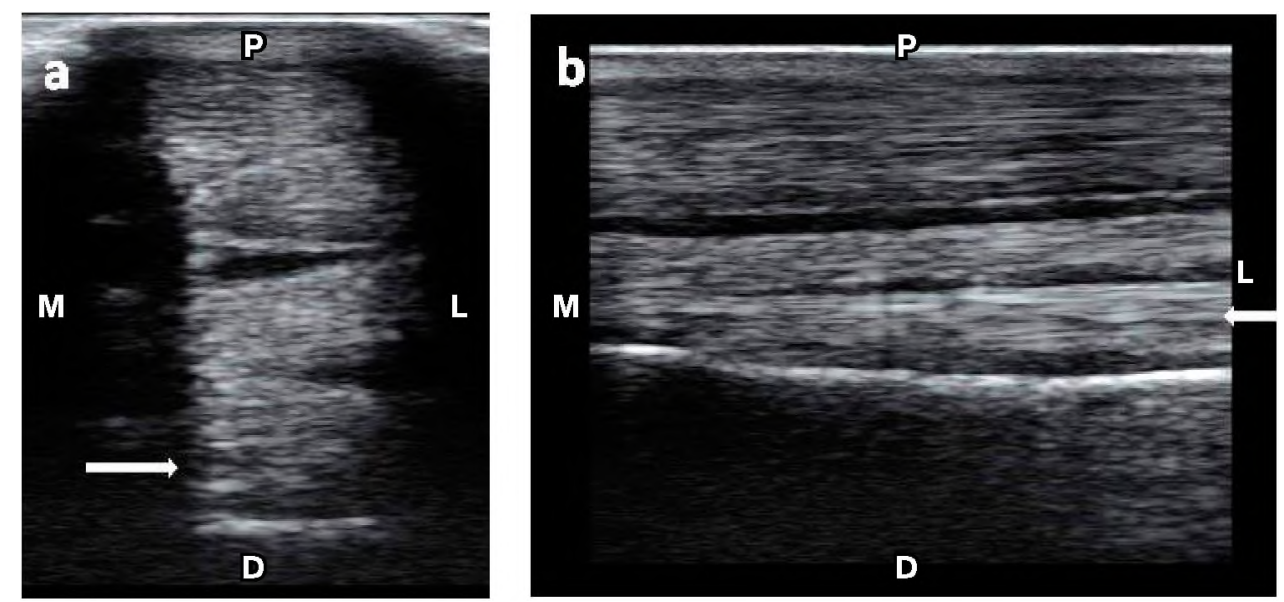

Figura 1. Imágenes de US axial (a) y sagital (b) del LS (flecha) en la región metacarpiana proximal (M: medial; L: lateral; P: proximal; D: distal). 
Tabla 1. Espesor dorso-palmar y latero-medial del origen del ligamento suspensorio obtenido en equinos mediante US.

\begin{tabular}{lcc}
\hline miembro & $\begin{array}{c}\text { miembro } \\
\text { anterior derecho }\end{array}$ & $\begin{array}{c}\text { miembro } \\
\text { anterior izquierdo }\end{array}$ \\
\hline espesor dorso-palmar & $0,77 \pm 0,13 \mathrm{~cm}$ & $0,79 \pm 0,11 \mathrm{~cm}$ \\
espesor latero-medial & $1,12 \pm 0,14 \mathrm{~cm}$ & $1,19 \pm 0,16 \mathrm{~cm}$ \\
\hline
\end{tabular}

Tabla 2. Espesor dorso-palmar y latero-medial, obtenido mediante resonancia magnética, del origen del ligamento suspensorio del miembro anterior en equinos de trabajo.

\begin{tabular}{lcc}
\hline miembro & $\begin{array}{c}\text { miembro } \\
\text { anterior derecho }\end{array}$ & $\begin{array}{c}\text { miembro } \\
\text { anterior izquierdo }\end{array}$ \\
\hline espesor dorso-palmar & $0,83 \pm 0,18 \mathrm{~cm}$ & $0,67 \pm 0,08 \mathrm{~cm}$ \\
espesor latero-medial & $1,11 \pm 0,22 \mathrm{~cm}$ & $1,18 \pm 0,24 \mathrm{~cm}$ \\
\hline
\end{tabular}

tejido adiposo y muscular otorgó una apariencia moteada o de puntillado, creando imágenes heterogéneas. El paquete vasculo-nervioso, vena-arteria-nervio metacarpiano palmar, se ubicó en medial de dicha región. En los cortes longitudinales se observó alineamiento fibrilar del ligamento.

En la Tabla 1 se expresa el espesor dorso-palmar y latero-medial del origen del LS obtenido mediante US. El LS adoptó una forma bilobulada en imágenes de RM axial-transversal (Figura 2a). La periférica de cada lóbulo se expresó con baja señal de intensidad (negro) correspondiente a las fibras ligamentosas y una zona central con alta señal (blanco) correspondiente a las fibras musculares y tejido adiposo.

En la imagen coronal-dorsal, el tejido muscular y adiposo, se expresó con imágenes lineales verticales, con señal intermedia a alta. En registros sagital-longitudinal el LS adoptó un aspecto de banda lisa y uniforme, paralela al hueso metacarpo III, mientras que los tejidos muscular y adiposo presentaron intensidad de señal intermedia a alta (Figura 2b).

El tejido conectivo que rodea al LS tuvo una intensidad de señal intermedia a alta, en todas las secuencias de pulso (T1, T2 y densidad protónica). El paquete vasculo-nervioso, vena-arteria-nervio metacarpiano/a palmar, se ubicó medial al LS como áreas focales o difusas de señal baja y heterogénea, en comparación al tejido conectivo.

La Tabla 2 presenta el espesor dorso-palmar y latero-medial del origen del LS obtenido mediante RM.

\section{DISCUSIÓN}

Las imágenes del LS mediante US expresan ecogenicidad heterogénea por la composición y forma de los diferentes tejidos que lo componen (músculo, grasa y tejido conectivo) en coincidencia con lo descrito por otros autores ${ }^{1}$. Según otros ${ }^{12,19-20}$, las propiedades acústicas de los diferentes tejidos son las que determinan esa variación en la ecogenicidad.

El tejido muscular tiene menor ecogenicidad que las fibras del ligamento, provocando una variación en los patrones ecográficos normales del LS. El tejido graso presentó una mayor ecogenicidad, la cual puede ser similar a las fibras del ligamento. Por otro lado, la ecogenicidad del tejido muscular depende de la angulación de la sonda de US, en contraste con lo que ocurre con el tejido graso.

El LS adoptó una forma rectangular en el corte axial ${ }^{19,20}$ y en la imagen longitudinal apareció una ligera concavidad en la disposición de sus fibras, similar a lo descrito por otros investigadores ${ }^{3}$. Las dimensiones del espesor dorso-palmar y latero-medial del LS fueron similares a las reportadas por otros autores para equinos deportivos ${ }^{1,19,20}$

En las imágenes obtenidas con RM, el LS adoptó una forma bilobulada al corte axial coincidente a lo

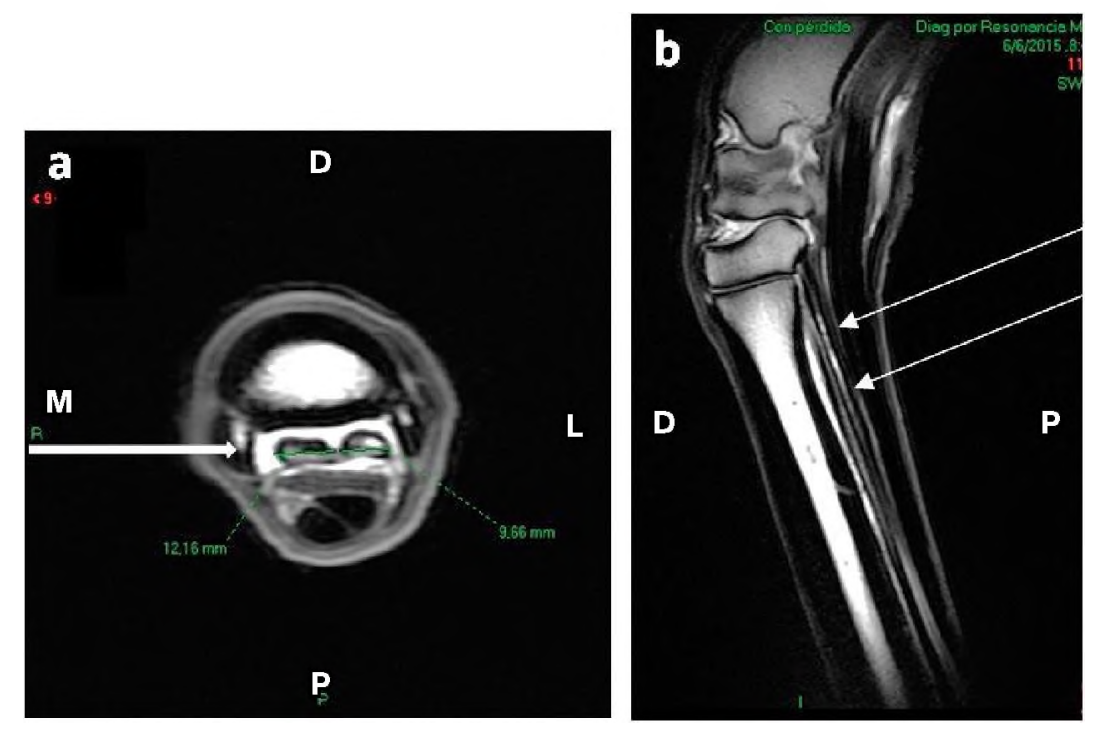

Figura 2. Imágenes de RM del origen del LS (flechas) en plano axial (a) a $2 \mathrm{~cm}$ distal de la articulación carpometacarpiana y sagital (b). M: medial. L: lateral; D: dorsal, P: palmar. 
descrito por otros autores $10,11,19,20$. El área central de cada lóbulo, conformado por tejido adiposo y muscular, generó alta señal de intensidad, mientras que la periferia presentó baja señal debido a las fibras ligamentosas, como lo propusieron varios investigadores ${ }^{2,10,11}$.

Independientemente del tipo de secuencia de imágenes y planos utilizados en RM, es importante reconocer la variable apariencia del aspecto proximal del LS en la cantidad, forma y localización de tejido muscular y adiposo dentro del ligamento en equinos sanos. Estos hallazgos coinciden con los resultados obtenidos en caballos deportivos ${ }^{10,11}$

Por lo tanto, la presencia de alta señal para el tejido ligamentoso y baja señal para el tejido muscular y adiposo, deben ser consideradas como signo de lesión del LS, de acuerdo con investigadores ${ }^{2}$.

En conclusión, el ultrasonido (US) y la resonancia magnética (RM) permitieron evaluar la constitución tisular variada del origen del ligamento suspensorio (LS) en miembros anteriores de equinos de trabajo asintomáticos y determinar medidas promedio de espesor que concuerdan con aquellas halladas en caballos deportivos, según la bibliografía consultada.

Agradecimientos. A las entidades que brindaron apoyo económico. A los propietarios, familia Villa y personal del establecimiento acopiador de equinos (Basail, Argentina). Al Vet. Miguel Rébora y personal del Frigorífico Lamar, Mercedes, Buenos Aires.

\section{REFERENCIAS}

1. Bischofberger $\mathbf{M}$ et al. 2006. Magnetic resonance imaging, ultrasonography and histology of the suspensory ligament origin: a comparative study of normal anatomy of Warmblood horses. Equine Vet J 38: 508-516.

2. Brokken MT et al. 2007. Magnetic resonance imaging features of proximal metacarpal and metatarsal injuries in the horse. Vet Radiol Ultrasound 48: 6, 507-517.

3. Denoix JM, Coudry V, Jacquet S. 2008. Ultrasonographic procedure for a complete examination of the proximal third interosseous muscle (proximal suspensory ligament) in the equine forelimbs. Equine Vet Educ 20: 3, 148-153.

4. Dyson S. 2007. Diagnosis and management of common suspensory lesions in the forelimbs and hind limbs of sport horses. Clin Tech Equine Pract 6: 179-188.

5. Dyson S. 2007. Proximal suspensory desmitis in the forelimbs and the hind limb. Proceedings of the Annual Convention of the AAEP 46: 137-142.
6. Dyson S. 2007. Proximal suspensory desmitis in the sports horse in forelimbs. American Association of Equine Practitioners. Br Vet J 150: 3, 279-291.

7. Dyson S. 1991. Proximal suspensory desmitis: clinical, ultrasonographic and radiographic features. Equine Vet $J 23$ : 1, 25-31.

8. Gibson KT, Steel CM. 2002. Conditions of the suspensory ligament causing lameness in horses. Equine Vet Educ 14: $1,39-50$.

9. Murray RC, Dyson S. 2007. Image interpretation and artifacts. Clin Tech Equine Practice 6: 16-25.

10. Nagy A, Dyson S. 2009. Magnetic resonance anatomy of the metacarpal region of the horse described from images acquired from low and high field magnets. Vet Radiol $\mathrm{Cl}$ trasound 50: 6, 595-605.

11. Nagy A, Dyson S. 2012. Magnetic resonance imaging and histological findings in the proximal aspect of the suspensory ligament of forelimbs in non lame horses. Equine Vet J 44: 43-50.

12. Pharr JW, Nyland TG. 1984. Sonography of the equine palmar metacarpal soft tissues. Vet Radiol 25: 6, 265-273.

13. Rantanen N. 2008. Imaging is believing-musculoskeletal ultrasonography. Compendium Equine 3: 7, 356-364.

14. Sampson SN, Tucker RL. 2007. Magnetic resonance imaging of the proximal metacarpal and metatarsal regions. Clin Tech Equine Pract 6: 78-85

15. Schramme M, Josson A, Linder K. 2012. Characterization of the origin and body of the normal equine rear suspensory ligament using ultrasonography, magnetic resonance imaging and histology. Vet Radiol Ultrasound 53: 3 , 318-328.

16. Sisson S, Grossman JD. 1959. Sindesmología general (artrología). En: Anatomía de los Animales Domésticos, $5^{\circ}$ ed., Salvat, Barcelona, p.399-407.

17. Stashak TS. 2004. Anatomía funcional de los órganos locomotores del caballo. En: Adams: claudicación en el caballo, $3^{\circ}$ Ed., Intermédica, Buenos Aires, p.24-30.

18. Stashak TS. 2004. Imágenes diagnósticas en caballos. En: Adams: Claudicación en el caballo, $3^{\circ} \mathrm{ed}$., Intermédica, Buenos Aires, p.315-347.

19. Werpy NM, Denoix JM. 2012. Imaging of the equine proximal suspensory ligament. Vet Clin Equine 28: 507 525.

20. Werpy NM, Denoix JM, McIlwraith CW, Frisbie DD. 2013. Comparison between standard ultrasonography, angle contrast ultrasonography, and magnetic resonance imaging characteristics of the normal equine proximal suspensory ligament. Vet Radiol Ultrasound 54: 5, 536547. 